\title{
Skin rash in patients treated with neoadjuvant erlotinib (Tarceva) in resectable non-small cell lung cancer: Predictor for tumor response and survival?
}

\author{
M.H. van Gool' ${ }^{1}$ J.A. Burgers², K. Sikorska ${ }^{3}$, E.E. Schaake², T.S. Aukema ${ }^{4}$, H.M. Klomp ${ }^{1, *}$ and on behalf of the NEL study Group \\ 1 Department of Surgical Oncology, The Netherlands Cancer Institute - Antoni van Leeuwenhoek Hospital, Amsterdam \\ 2 Department of Thoracic Oncology, The Netherlands Cancer Institute - Antoni van Leeuwenhoek Hospital, Amsterdam \\ ${ }^{3}$ Department of Biometrics, The Netherlands Cancer Institute - Antoni van Leeuwenhoek Hospital, Amsterdam \\ ${ }^{4}$ Department of Nuclear Medicine, The Netherlands Cancer Institute - Antoni van Leeuwenhoek Hospital, Amsterdam
}

\begin{abstract}
Background: Skin rash during treatment with epidermal growth factor receptor (EGFR)-tyrosine kinase inhibitors (TKI) has been reported to be predictive for response and survival in patients with advanced non-small cell lung cancer (NSCLC). The aim of this analysis was to evaluate whether skin rash during treatment (as a biomarker) in a preoperative setting was related to response and survival. Methods: This study was designed as an open-label phase II trial (also known as M06NEL). Patients received preoperative erlotinib (Tarceva) $150 \mathrm{mg}$ once daily for 3 weeks. Skin toxicity during treatment was analysed in relation to metabolic and histopathological response, overall survival (OS) and progression-free survival (PFS). Results: In total 59 patients (25 male, 34 female) were eligible for analysis. In 39 patients (66\%) skin toxicity occurred. According to National Cancer Institute Common Toxicity Criteria (NCICTC), Grade 1 toxicity was seen in 15 patients (25\%), Grade 2 in 19 patients (32\%) and Grade 3 in five patients (8\%). None of the patients showed skin toxicity Grade 4 and 5. The median follow up was 74 months. Thirty-six patients (61\%) were alive at time of analysis. Twenty-seven patients (46\%) showed disease progression during follow up. Hazard ratios (HR) indicated lower risk of death $(\mathrm{HR}=0.66,95 \% \mathrm{Cl}$ : 0.29 $1.50)$ and progression $(H R=0.64,0.30-1.36)$, although in this small group results were not significant. Skin rash did not adequately predict response. Conclusions: In this neoadjuvant setting with limited treatment time in patients with early stage NSCLC, skin rash was not associated with response and survival and cannot be used as an early biomarker.
\end{abstract}

Keywords: skin rash; neoadjuvant erlotinib; resectable; non-small cell lung cancer; tumor response; survival

\section{Introduction}

Targeted agents such as epidermal growth factor receptor (EGFR)-tyrosine kinase inhibitors (TKI) have been become part of standard practice for the treatment of advanced non-small cell lung carcinoma (NSCLC). EGFR is a transmembrane glycoprotein which is expressed in many normal cells of epithelial origin, playing a role in cell growth and differentiation [1]. EGFR is overexpressed or harbors activating mutations in the majority of NSCLCs and in this form acts as a key regulator of tumor cell proliferation, angiogenesis, invasion, and metastasis [2]. EGFR-TKIs, such as erlotinib (Tarceva), are reversible binders to the EGFR tyrosine kinase, competing with the substrate, and can block the catalytic activity of the enzyme [3].

Compared with traditional chemotherapy, the severity of the associated toxicity with EGFR-TKI therapy is mild [4]. However many patients develop a dermatologic reaction, particularly a papulopustular rash (Figure 1) [5]. This skin toxicity can be explained by an alteration of EGFRs function of basal keratinocytes of the skin [6]. Skin rash in response to EGFR-TKI therapy may be an expression of the EGFR-TKI therapeutic effect on tumors, although the

\footnotetext{
*Corresponding author: Houke M Klomp, MD, PhD., Department of Surgical Oncology, The Netherlands Cancer Institute - Antoni van Leeuwenhoek Hospital, Plesmanlaan 121, 1066 CX Amsterdam, The Netherlands. Fax: +31 20 5122554; Email: h.klomp@nki.nl

Received 9 June 2017 Revised 14 July 2017 Accepted 21 July 2017 Published 29 July 2017

Citation: van Gool MH, Burgers JA, Sikorska K, Schaake EE, Aukema TS, Klomp HM, NEL study Group. Skin rash in patients treated with neoadjuvant erlotinib (Tarceva) in resectable non-small cell lung cancer: Predictor for tumor response and survival? J Cancer Res Ther. 2017; 5(7):45-49. DOI: 10.14312/2052-4994.2017-9

Copyright: (c) 2017 van Gool MH, et al. Published by NobleResearch Publishers. This is an open-access article distributed under the terms of the Creative Commons Attribution License, which permits unrestricted use, distribution and reproduction in any medium, provided the original author and source are credited.
} 
exact mechanism of the association between skin rash and EGFR-TKI efficacy is not elucidated.

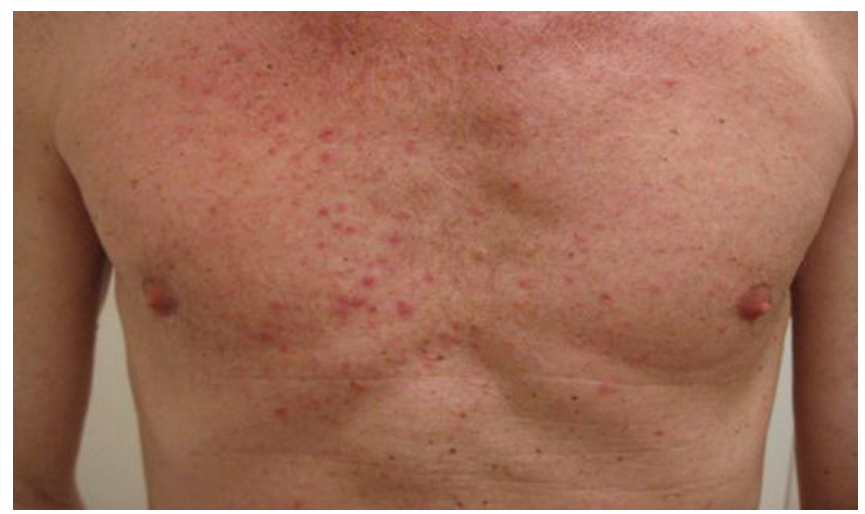

Figure 1 Patient with papulopustular rash. Mild skin rash after 10 days of erlotinib (Tarceva) treatment.

In advanced stage NSCLC, an association of clinical benefit with skin toxicity has been found. Although there is conflicting data about this association, a meta-analysis by Liu et al. showed that skin rash after using EGFR-TKI was an efficient clinical marker for predicting response of patients with advanced NSCLC to EGFR-TKIs. Furthermore, patients with a skin rash had a longer PFS and OS [6-10].

The M06NEL phase II study was designed to evaluate response of a short course of neoadjuvant (preoperative) erlotinib in patients with early stage NSCLC $[11,12]$. This study included patients with unknown EGFR mutation status from 2006 - 2010, and currently mature survival data are available. Response data have been published before [11]. In the light of discussions on skin toxicity as a "biomarker", the aim of this analysis was to evaluate whether skin toxicity during preoperative treatment was related to response and survival.

\section{Materials and methods}

\section{Study design}

The design, eligibility criteria and treatment schedule have been described in detail elsewhere [11]. In short, this study was designed as an open-label, non-comparative phase II study performed in four hospitals in The Netherlands and was approved by each local independent ethics committee and Institutional Review Board (IRB or equivalent). All patients signed an Informed Consent Form.

Patients with newly diagnosed resectable NSCLC, i.e., clinical T1-3 N0-1, were allowed to enter the study. The primary lesion had to be measurable, i.e., the longest diameter being $\geq 1 \mathrm{~cm}$ measured by spiral CT scan. Sixty patients received one tablet of $150 \mathrm{mg}$ erlotinib daily during an intended course of three weeks. Surgical resection was scheduled in the fourth week after start of treatment. Patients who received erlotinib for at least one day were included in this analysis.

\section{Severity grading}

Skin rash was assessed after one and three weeks of erlotinib therapy. US National Cancer Institute Common Toxicity criteria (NCICTC version 4.0) was used to grade severity of skin toxicity. Grade 1: Papules and/or pustules covering < $10 \%$ Body Surface Area (BSA), which may or may not be associated with symptoms of pruritus or tenderness, Grade 2: Papules and/or pustules covering 10 - 30\% BSA, which may or may not be associated with symptoms of pruritus or tenderness; associated with psychosocial impact; limiting instrumental ADL, Grade 3: Papules and/ or pustules covering $>30 \%$ BSA, which may or may not be associated with symptoms of pruritus or tenderness; limiting self-care ADL; associated with local super infection with oral antibiotics indicated, Grade 4: Papules and/ or pustules covering any \% BSA, which may or may not be associated with symptoms of pruritus or tenderness and are associated with extensive superinfection with IV antibiotics indicated; life threatening consequences, and Grade 5: Death [9]. For analysis purposes, skin toxicity (dichotomically) was defined as either absence or presence of skin toxicity (Grade 1 or higher).

\section{Response data and follow up}

Metabolic response

PET/CT scan imaging was performed using a hybrid system (GeminiTF, Philips, Eindhoven, The Netherlands) 60 min after fludeoxyglucose (FDG) injection. 18F-FDG was administered in dosages of 180 - 240 megabecquerel (MBq). Patients fasted for 6 hours prior to imaging. Diabetes mellitus was regulated in advance, with plasma glucose < $10 \mathrm{mmol} / \mathrm{l}$. The interval between FDG administration and scanning was 60 minutes +/- 10 min. Low-dose CT images (40 mAs, $5 \mathrm{~mm}$ slices) were acquired without intravenous contrast.

The images generated (PET/CT, low dose CT and PET) were displayed using an OsirixDicom viewer in a Unixbased operating system (Mac Pro, Apple, Cupertino, CA, USA) and were evaluated on the basis of two-dimensional orthogonal reslicing. The images were evaluated by one nuclear physician (R.V.O.). A baseline FDG-PET/CT scan was obtained during routine staging in all patients. The baseline FDG-PET/CT scan had to be acquired within one month before the start of erlotinib treatment. For metabolic response monitoring, FDG-PET/CT scan was planned around 21 days after the initiation of erlotinib therapy. All metabolic data were compared to the individual's baseline data.

All imaging data were sent to The Netherlands Cancer Institute - Antoni van Leeuwenhoek Hospital (NKI-AVL) for central review and analyses. FDG-PET/CT scans performed during and after treatment with erlotinib were compared to baseline FDG-PET/CT scans. PET/CT imaging was only evaluable when scans were acquired with the same scanner, acquisition protocol, and reconstruction software, and with similar intervals from tracer injection to scanning. FDG tumour uptake was quantified using SUVmax (maximum activity concentration of FDG divided by the injected dose and corrected for the body weight of the patient). For the determination of the SUVmax, the maximum FDG-uptake was searched within the volume of the primary tumour. These regions of interest (ROI) were manually drawn. Metabolic response classification was assessed following the European Organization for Research and Treatment of Cancer (EORTC) criteria for tumour response [13]. In short, progressive metabolic disease was classified as an increase 
in SUVmax by more than $+25 \%$. Stable metabolic disease as an increase or a decrease of SUVmax by less than $+25 \%$ or $-25 \%$, and partial metabolic response as a SUVmax reduction by at least $-25 \%$. For this analysis, patients with complete or partial metabolic response were defined as "responders" and patients with stable metabolic disease and progressive metabolic disease were defined as "nonresponders".

\section{Histopathological response}

The resection specimens were scored for residual vital tumour and the presence of morphological signs of therapy-induced regression such as necrosis with foam cell reaction, giant cell reaction, cholesterol clefts and fibrotic alterations (Junker classification [14]). For reporting in this study, a cut off of $50 \%$ necrosis (with morphological signs of therapy-induced regression) was used.

Mutation testing was performed centrally at the certified laboratory of the NKI-AVL. Tumours were tested for EGFR mutation.

\section{Follow up}

Follow up was measured from the start of erlotinib treatment and available through September 2015 at the time of analysis, which guaranteed a minimal follow up of five years. The primary endpoint of this study was response. One of the secondary endpoints was overall survival (OS). Overall survival was defined as the time from the date of start of erlotinib treatment to the date of all-cause death or to the last day of follow up. Another secondary endpoint was progression-free survival (PFS). Progression-free survival was defined as the interval between the start of erlotinib treatment and the earliest occurrence of disease progression, either loco regional recurrence or distant dissemination, or death from any cause [15]. Clinical follow up visits took place every three months during the first year after treatment and then twice every year for at least the following five years. During clinical follow up visits, performance status was assessed, patients underwent a physical exam and both a chest radiograph and blood samples (complete blood cell count, and metabolic panel) were taken. Minimum follow up of five years was available for all patients.

\section{Statistical analyses}

Association between skin rash (any Grade rash versus no rash) and response was tested using the Fisher exact test described as the probability of response ratio (PRR). Survival curves were derived using the Kaplan-Meier method and compared with log-rank test. Hazard ratios and $95 \% \mathrm{Cl}$ were obtained from the proportional hazard Cox model. All analyses were performed with $\mathrm{R}$ (version 3.2.3) and SPSS 22.

\section{Results}

From December 2006 until November 2010, 60 patients were enrolled in this study. Patient flow diagram is shown in Figure 2. In total 59 patients ( 25 male, 34 female) received at least 1 day of erlotinib and were eligible for this analysis. Patients received median treatment of 20 days (range 5-28 days). Median age was 64 years (range 36 - 76). Patient

\section{Skin toxicity}

In 39 (66\%) patients skin toxicity occurred. According to US National Cancer Institute catalogue of common toxicity criteria (NCICTC) Grade 1 was seen in 15 patients (25\%),

\begin{tabular}{|c|c|}
\hline $\begin{array}{l}\text { Inclusion } \\
\text { Malignancy (NSCLC) proven } \\
\text { Malignancy (NSCLC) highly probable }\end{array}$ & $\begin{array}{l}(n=60) \\
(n=56) \\
(n=4)\end{array}$ \\
\hline $\begin{array}{l}\text { Erlotinib (Tarceva) treatment } \\
\text { Full } 21 \text {-day } \\
\text { 15-21 days } \\
1-15 \text { days }\end{array}$ & $\begin{array}{l}(n=59) \\
(n=42) \\
(n=9) \\
(n=8)\end{array}$ \\
\hline Metabolic response assessment & $(n=58)$ \\
\hline Histopathological response assessment & $(n=59)$ \\
\hline Follow up data available & $(n=59)$ \\
\hline
\end{tabular}

Figure 2 Patient flow diagram.

Table 1 Patients characteristics.

\begin{tabular}{|c|c|c|}
\hline & $n=59$ & $\% /$ range \\
\hline Gender M/F & $25 / 34$ & $42 / 58$ \\
\hline Mean age at diagnose & 64 & $36-76$ \\
\hline \multicolumn{3}{|l|}{ Smoking status } \\
\hline Never & 14 & $24 \%$ \\
\hline Former & 26 & $44 \%$ \\
\hline Current & 19 & $32 \%$ \\
\hline \multicolumn{3}{|l|}{ Clinical stage } \\
\hline IA & 18 & $30 \%$ \\
\hline IB & 14 & $24 \%$ \\
\hline$\| \mathrm{A}$ & 4 & $7 \%$ \\
\hline IIB & 4 & $7 \%$ \\
\hline$\geq \mathrm{IIIA}$ & 19 & $32 \%$ \\
\hline \multicolumn{3}{|l|}{ Mutation status } \\
\hline EGFR & 6 & $10 \%$ \\
\hline
\end{tabular}

characteristics of this group are listed in Table 1. Complete toxicity data were described before; in short toxicity of this group is listed in Table 2 [11]. 


\begin{tabular}{lcccccc}
\hline Table 2 Toxicity. & \multicolumn{1}{l}{ Trade 1} & Grade 2 & Grade 3 & Grade 4 & Grade 5 & Total \\
\hline Rash & 15 & 19 & 5 & 0 & 0 & 39 \\
Diarrhea & 13 & 6 & 2 & 0 & 0 & 21 \\
Dry skin & 9 & 4 & 0 & 0 & 0 & 13 \\
Dry eyes & 0 & 8 & 0 & 0 & 0 & 8 \\
Pruritus & 2 & 5 & 0 & 0 & 0 & 7 \\
Fatigue & 6 & 0 & 1 & 0 & 0 & 7 \\
$\begin{array}{l}\text { Nausea/ } \\
\text { vomiting }\end{array}$ & 4 & 0 & 0 & 0 & 0 & 4 \\
Mucositis & 1 & 3 & 0 & 0 & 0 & 4 \\
$\begin{array}{l}\text { Infection/ } \\
\text { pneumonitis }\end{array}$ & 0 & 2 & 1 & 0 & 0 & 3 \\
Anorexia & 1 & 1 & 0 & 0 & 0 & 2 \\
Blurry vision & 1 & 0 & 0 & 0 & 0 & 1 \\
\hline
\end{tabular}

Note: Complete toxicity data is described elsewhere [11].

Grade 2 in 19 patients (32\%), Grade 3 in 5 patients (8\%). None of the patients showed skin toxicity Grade 4 and 5.

\section{Response according to skin toxicity}

In 58 patients both FDG-PET scans were available to assess metabolic response. In 16 patients (28\%), metabolic response was seen according to the EORTC criteria. The metabolic response rate in the skin toxicity group was $29 \%$ versus $25 \%$ in the no skin toxicity group. The association test showed no relation between skin rash and metabolic response of the tumour (PRR $0.86(95 \% \mathrm{Cl}: 0.35-2.14)$ $p=0.99$ ) (Table 3). In 59 patients surgical specimen was available for assessment of histopathological response of the tumour. According to the Juncker classification in 12 patients (20\%) a histopathological response was seen. The histopathological response rate in the skin toxicity group was $21 \%$ versus $20 \%$ in the no skin toxicity group (Table 3 ). The association test showed no relation between rash and histopathological response of the tumour (PRR 0.98 (95\% $\mathrm{Cl}: 0.33-2.85) \mathrm{p}=0.99$ ). Exploratory analyses with grading of skin toxicity did not show any differences either. In 6 patients (10\%) an EGFR mutations was found. Mutations in the EGFR gene did not predict the occurrence of rash ( $p$ $=0.65)$.

Table 3 Response.

\begin{tabular}{lcccc}
\hline & \multicolumn{2}{c}{ Metabolic response } & \multicolumn{2}{l}{ Histopathological response } \\
\hline yes & no & yes & no \\
Skin toxicity & 11 & 27 & 8 & 31 \\
No skin toxicity & 5 & 15 & 4 & 16 \\
\hline
\end{tabular}

\section{Survival according to skin toxicity}

The median follow-up was 74 months. Thirty-six patients $(61 \%)$ were alive at time of analysis. Twenty-seven patients (46\%) showed disease progression after treatment. Thus, median OS and PFS were not reached. The five-year overall survival was 64\% (95\% Cl: 53 - 78\%) and the five-year PFS was 56\% (44 - 70\%). Kaplan-Meier curves for both end points are displayed in Figures $3 a$ and $3 b$.
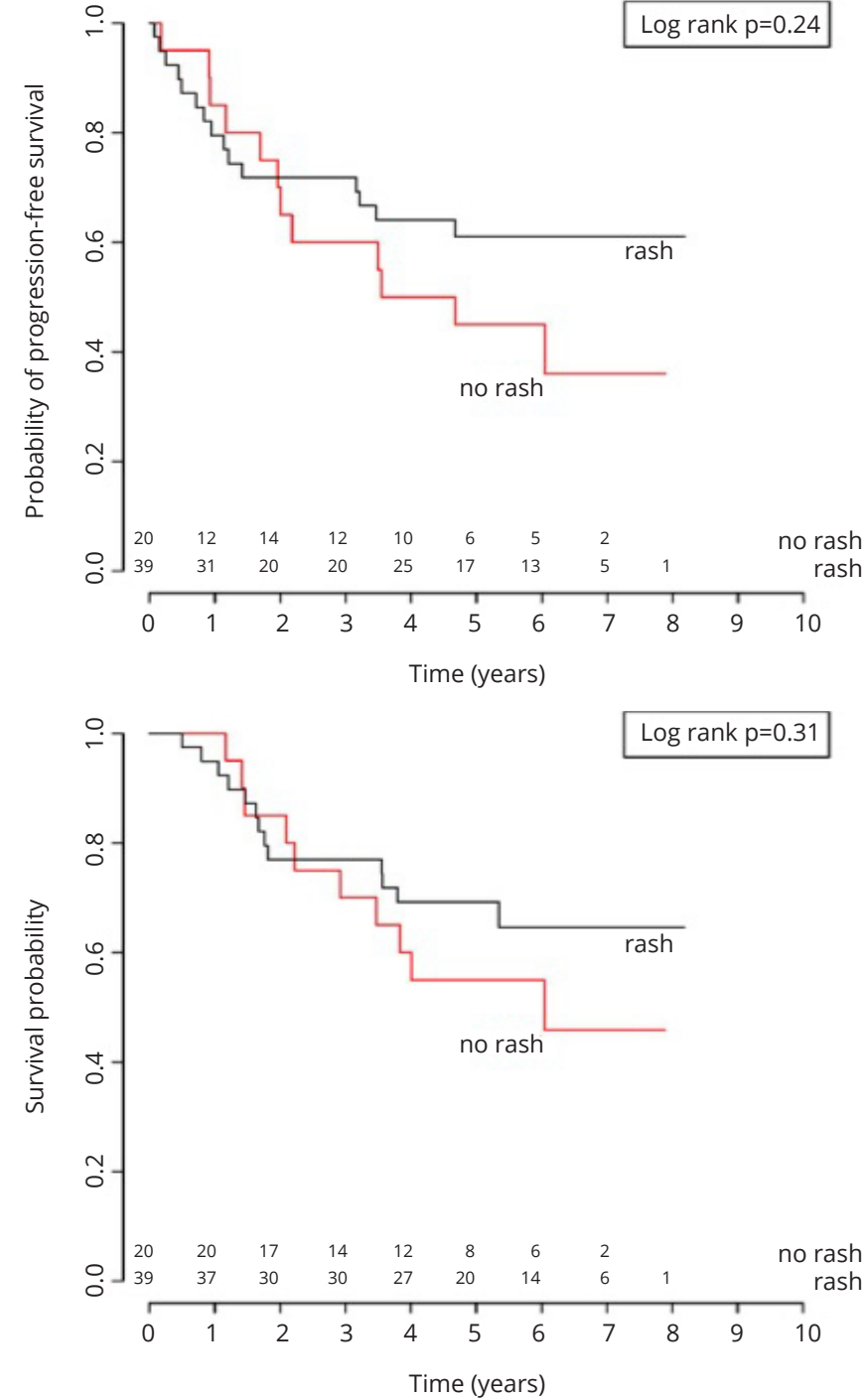

Figure 3 Kaplan-Meier curves for overall survival (a) and progression-free survival (b) for patients according to skin rash.

In the univariable Cox models, the trend for patients with skin rash showed lower risk for progression $(H R=0.64$, 95\% Cl: $0.30-1.36, p=0.25)$ and death $(H R=0.66,95 \%$ $\mathrm{Cl}: 0.29-1.5, \mathrm{p}=0.32$ ), but these hazard ratios were not statistically significant. Exploratory analyses with grading of skin toxicity did not add extra information.

\section{Discussion}

Three weeks of EGFR-TKI treatment in patients with early stage NSCLC resulted in skin rash in the majority (66\%), mostly mild to moderate in severity. Although presence of skin toxicity may be associated with superior (disease-free) survival, skin rash as a "biomarker" was not adequate as a predictor for early response and survival.

In a meta-analysis, Lui et al. reported on the relation between skin rash during EGFR-TKI and response and survival. In this meta-analysis, all trials reporting on rash or the absence of rash and survival showed significant associations of skin rash with favorable survival. They showed that the risk of death decreased by $60 \%$ in patients with rash as compared to patients without rash $(\mathrm{HR}=0.40$, 95\% Cl: $0.28-0.52 p=<0.01)$. For PFS they showed that the risk of disease progression for patients treated with a 
TKI with rash decreased by $55 \%$ as compared to patients without a rash $(\mathrm{HR}=0.45,95 \% \mathrm{Cl}: 0.37-0.53 \mathrm{p}=<0.01)$ [10]. In our study, the predictive or prognostic value of skin rash as a 'biomarker' was not significant. This may be mainly due to short treatment time and the Stage of disease.

Skin rash is a main side effect of EGFR-TKI therapy and occurs in approximately two-thirds of patients with NSCLC [6]. Similar prevalence of skin rash was observed in our study. Skin toxicity is rarely lethal or severely disabling, but it may cause social impairment and lead to discontinuation or dose modification of EGFR-TKI's [16]. Encouraging patients suffering from skin rash during (neoadjuvant) EGFR-TKI treatment to continue treatment could potentially benefit survival. Even in our study with short treatment duration, 20 patients (34\%) discontinued treatment prematurely; therefore data showing a predictive relation of skin rash to better outcome may help patients to better accept toxicity.

Skin rash in response to EGFR-TKI therapy may be an outward manifestation of the EGFR-TKI therapeutic effect on tumors. Although the presence of a sensitizing EGFR mutation has become paramount in EGFR-TKI therapy, it may be difficult to acquire genotypes of EGFR either due to tumor sample difficulties, logistic or financial reasons. Unfortunately, this study does not provide evidence that skin rash may be used as a cheap and effective biomarker for response.

Several limitations of this study must be acknowledged. The patient population includes a heterogeneous group of NSCLC subtypes. Still, classifying tumors in the preoperative setting, in small diagnostic samples acquired by percutaneous biopsy, is challenging by the paucity of tumor cells and the absence of tissue architecture [17]. However, diagnostic techniques have improved to evaluate mutation status and even next-generation-sequencing in small biopsies. Secondly, previous studies showed that rash develops within the first three to four weeks of treatment, and typically peaks in severity three to five weeks after treatment initiation $[7,18]$. In our population the maximum period of treatment was three weeks, therefore skin rash may be underestimated. Supportive treatment may have decreased severity and appearance of skin toxicity, which potentially hampers our analysis $[19,20]$.

Furthermore, patients included in this study all had a relatively small burden of disease compared to Stage IV NSCLC patients described in other studies. The extent of disease could also be a factor influencing skin rash.

\section{Conclusion}

In our series skin rash did not adequately predict response and survival. Therefore skin rash cannot be used as an early "biomarker" in this setting. Recently developed test, for example "fluid phase biopsy" and "targeted nextgeneration" sequencing could provide new options to predict response during EGFR-TKI therapy [21, 22].

\section{Conflict of interest}

This phase II study was an investigator-initiated study, supported by an unrestricted educational grant from Roche, the Netherlands.

\section{References}

[1] Carpenter G, Cohen S. Epidermal growth factor. J Biol Chem. 1990; 265(14):7709-7712.

[2] Soria JC, Mok TS, Cappuzzo F, Janne PA. EGFR-mutated oncogeneaddicted non-small cell lung cancer: current trends and future prospects. Cancer Treat Rev. 2012; 38(5):416-430.

[3] Harari PM, Allen GW, Bonner JA. Biology of interactions: Antiepidermal growth factor receptor agents. J Clin Oncol. 2007; 25(26):4057-4065.

[4] West HL, Franklin WA, McCoyJ, Gumerlock PH, Vance R, et al. Gefitinib therapy in advanced bronchioloalveolar carcinoma: Southwest Oncology Group Study S0126. J Clin Oncol. 2006; 24(12):1807-1813.

[5] Chanprapaph K, Vachiramon V, Rattanakaemakorn P. Epidermal growth factor receptor inhibitors: A review of cutaneous adverse events and management. Dermatol Res Pract. 2014; 2014:734249.

[6] Lacouture ME. Mechanisms of cutaneous toxicities to EGFR inhibitors. Nat Rev Cancer. 2006; 6(10):803-812.

[7] Perez-Soler R, Chachoua A, Hammond LA, Rowinsky EK, Huberman $M$, et al. Determinants of tumor response and survival with erlotinib in patients with non-small-cell lung cancer. J Clin Oncol. 2004; 22(16):3238-3247.

[8] Zhou CC, Wu YL, Chen GY, Feng JF, Liu XQ, et al. Erlotinib versus chemotherapy as first-line treatment for patients with advanced EGFR mutation-positive non-small-cell lung cancer (OPTIMAL, CTONG-0802): A multicentre, open-label, randomised, phase 3 study. Lancet Oncology. 2011; 12(8):735-742.

[9] National Cancer Institute. Common terminology criteria for adverse events (CTCAE). U.S. Dept. of Health and Human Services, National Institutes of Health, National Cancer Institute. 2009.

[10] Liu HB, Wu Y, Lv TF, Yao YW, Xiao YY, et al. Skin rash could predict the response to EGFR tyrosine kinase inhibitor and the prognosis for patients with non-small cell lung cancer: A systematic review and meta-analysis. PLoS One. 2013; 8(1):e55128.

[11] Schaake EE, Kappers I, Codrington HE, Valdes Olmos RA, Teertstra $\mathrm{HJ}$, et al. Tumor response and toxicity of neoadjuvant erlotinib in patients with early-stage non-small-cell lung cancer. J Clin Oncol. 2012; 30(22):2731-2738.

[12] van Gool MH, Aukema TS, Schaake EE, Rijna $H$, Codrington $H E$, et al. (18)F-fluorodeoxyglucose positron emission tomography versus computed tomography in predicting histopathological response to epidermal growth factor receptor-tyrosine kinase inhibitor treatment in resectable non-small cell lung cancer. Ann Surg Oncol. 2014; 21(9):2831-2837.

[13] Young H, Baum R, Cremerius U, Herholz K, Hoekstra O, et al. Measurement of clinical and subclinical tumour response using [18F]fluorodeoxyglucose and positron emission tomography: Review and 1999 EORTC recommendations. European Organization for Research and Treatment of Cancer (EORTC) PET Study Group. Eur J Cancer. 1999; 35(13):1773-1782.

[14] Junker K, Thomas M, Schulmann K, Klinke F, Bosse U, et al. Tumour regression in non-small-cell lung cancer following neoadjuvant therapy. Histological assessment. J Cancer Res Clin Oncol. 1997; 123(9):469-477.

[15] Fumagalli D, Bedard PL, Nahleh Z, Michiels S, Sotiriou C, et al. A common language in neoadjuvant breast cancer clinical trials: Proposals for standard definitions and endpoints. Lancet Oncol. 2012; 13(6):e240-248.

[16] Joshi SS, Ortiz S, Witherspoon JN, Rademaker A, West DP, et al. Effects of epidermal growth factor receptor inhibitor-induced dermatologic toxicities on quality of life. Cancer. 2010; 116(16):3916-3923.

[17] Field RW, Smith BJ, Platz CE, Robinson RA, Neuberger JS, et al. Lung cancer histologic type in the surveillance, epidemiology, and end results registry versus independent review. J Natl Cancer Inst. 2004; 96(14):1105-1107.

[18] Lynch TJ Jr, Kim ES, Eaby B, Garey J, West DP, et al. Epidermal growth factor receptor inhibitor-associated cutaneous toxicities: An evolving paradigm in clinical management. Oncologist. 2007; 12(5):610-621.

[19] Pugliese SB, Neal JW, Kwong BY. Management of dermatologic complications of lung cancer therapies. Curr Treat Options Oncol. 2015; 16(10):50.

[20] Peuvrel L, Dreno B. Dermatological toxicity associated with targeted therapies in cancer: Optimal management. Am J Clin Dermatol. 2014; 15(5):425-444.

[21] Wendel M, Bazhenova L, Boshuizen R, Kolatkar A, Honnatti M, et al. Fluid biopsy for circulating tumor cell identification in patients with early-and late-stage non-small cell lung cancer: A glimpse into lung cancer biology. Phys Biol. 2012; 9(1):016005.

[22] HanJY, Kim SH, Lee YS, Lee SY, Hwang JA, et al. Comparison of targeted next-generation sequencing with conventional sequencing for predicting the responsiveness to epidermal growth factor receptortyrosine kinase inhibitor (EGFR-TKI) therapy in never-smokers with lung adenocarcinoma. Lung Cancer. 2014; 85(2):161-167. 